J. Jpn. Atheroscler. Soc. $20(8 \cdot 9)$ :

703-707, 1992

\title{
LDL Eliminates the Inhibitory Effect of Human Aortic Elastin on Cultured Rat Aortic Smooth Muscle Cell Migration In Vitro
}

\author{
Toshiro Ooyama*1, Hiroshi SAKAmoto*1, Katsunori FukudA*2, \\ Hiromi OdA ${ }^{* 3}$, Youko MuraI*4, Yoshiyuki SeYamA*5 and Hiroaki NaKAmura*6
}

\section{Introduction}

Elastin is the core protein of elastic fibers and plays a role in determining the elasticity of the arterial wall. Elastin is also thought to have biological effects on migration ${ }^{1)}$, growth ${ }^{2)}$, and phenotypic changes ${ }^{3)}$ in subcultured medial smooth muscle cells. The enhanced migration of cultured rat aortic smooth muscle cells (cultured rat aortic SMCs) in a Boyden chamber induced by rat platelet-derived attractant (rat PDA) is inhibited when the filter is coated with human aortic elastin ${ }^{1)}$.

Low density lipoprotein (LDL) is a plasma protein with a high affinity for the elastin ${ }^{4-6)}$ found deposited along with elastic fibers in atherosclerotic plaques? ${ }^{7}$. Thus, LDL may be involved in the inhibition of SMC migration induced by elastin as described above. However, few reports have addressed this point so far.

The present study was designed to study whether or not LDL acts on elastin coated filters. The filters onto which human aortic elastin was coated were treated with human plasma LDL or HDL and the effects of these pretreatments on the migration of cultured rat aortic SMCs toward rat PDA in a

*1 Division of Immunology, Tokyo Metropolitan Geriatric Hospital, Tokyo

*2 Department of Otorhynolaryngology, Kagoshima University, School of Medicine, Kagoshima

*3 Department of Orthopaedic Surgery, Faculty of Medicine, University of Tokyo, Tokyo

*4 Department of Respiratory Diseases, Tama Metropolitan Geriatric Hospital, Tokyo

*5 Department of Clinical Chemistry, Hoshi Pharmaceutical University, Tokyo

*6 Laboratory of Electron Microscopy, Saitama Medical University, Saitama

Received: November 1, 1991

Accepted: May 13, 1992
Boyden chamber were observed. The effect of the treating the filter with rabbit anti-human aortic elastin antibody was also studied.

\section{Materials and Methods}

Minimal essential medium (MEM) and fetal bovine serum (FBS) were from Gibco (Santa Clara, CA). Micropore filters $(0.22 \mu \mathrm{m}, 8 \mu \mathrm{m}$ pore size, $100 \mu \mathrm{m}$ thickness) were from Millipore Corporation (Bedford, MA).

\section{Preparation of Elastin Peptides and Collagens}

Elastin was prepared from human aorta as previously reported ${ }^{1)}$. Briefly, medial tissues from a normal aorta were washed and delipidated thoroughly with n-butanol-acetone and diethylether. The powdered, delipidated tissue was extracted with a solution of $2 \mathrm{M} \mathrm{MgCl}_{2}, 5 \mathrm{M}$ guanidine, and $0.1 \mathrm{M}$ mercaptoethanol in distilled water. The tissue was then washed with acetone and dried in a dessicator.

Elastin peptides, used for coating the filters and as an antigen for immunizing animals, was obtained by incubating the elastin powder in a solution of $1 \mathrm{M} \mathrm{KOH}$ in $80 \%$ ethanol with shaking at $37^{\circ} \mathrm{C}$ for 2 to 3 hours; this elastin preparation is referred to $\mathrm{k}$-elastin ${ }^{8}$.

$\mathrm{k}$-Elastin is heterogeneous in terms of molecular weight (10-300 kda) and has a similar amino acid composition to that in a previous publication ${ }^{9}$, although a relative decrease in glycine content was observed.

Type I collagen was prepared from human bone, and Types III and V from human placenta. Collagens were solubilized from these tissues by limited proteolysis with pepsin under nondenaturing conditions and purified.

Production of Rabbit Anti-Human Aortic Elastin Antibody

Anti-human aortic elastin antibody was produced in male Japanese rabbits ${ }^{10}$. One milliliter of $\mathrm{k}$ - 
elastin solution at a concentration of $5 \mathrm{mg} / \mathrm{m} l$ was emulsified with $1.25 \mathrm{ml}$ of Freund Complete Adjuvant (FCA).

One milliliter of k-elastin FCA emulsion containing approximately $2.25 \mathrm{mg}$ of k-elastin was injected in several places on the back skin and foot pads of the rabbits. Immunization was repeated at two week intervals for 2-3 months until the antibody titer increased. Ten days after the each final booster injection, blood was drawn from the animals.

The $\gamma$-globulin fraction was purified from rabbit anti-human aortic elastin antiserum by ammonium sulfate precipitation (50\% saturation) and DEAE cellulose chromatography. A control $\gamma$-globulin fraction was also isolated from normal rabbit serum by the same method. These $\gamma$-globulin fractions were exhaustively dialyzed against phosphate buffered

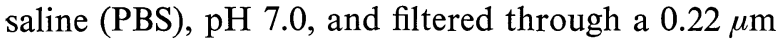
pore size micropore filter.

The cross-reactivity of the anti-elastin antibody to various types of collagen preparations (Types I, III, V) was tested by Western blotting. No cross reactive bands were found.

\section{Preparation of Low Density Lipoprotein and High Density Lipoprotein}

Lipoproteins from normal human plasma were fractionated by sequential flotation in a Beckman L3-50 preparative ultracentrifuge with a Type 50-Ti rotor, according to the standard technique ${ }^{11)}$ using $\mathrm{NaCl}$ for density adjustments. Low density lipoprotein (LDL) and high density lipoprotein (HDL) were isolated at solvent densities of 1.063 and 1.210, respectively. The isolated LDL and HDL were dialyzed exhaustively against $0.15 \mathrm{M} \mathrm{NaCl}$, containing $0.01 \%$ EDTA, $\mathrm{pH} 7.0$, at $4{ }^{\circ} \mathrm{C}$ and filtered through a $0.22 \mu \mathrm{m}$ pore size micropore filter. They were characterized chemically as well as by polyacrylamide gel electrophoresis.

\section{Coating of Micropore Filters with K-Elastin}

Micropore filters were coated with k-elastin as described previously ${ }^{1)}$; that is, the filters were immersed in a $0.5 \mathrm{M}$ acetic acid solution overnight at room temperature and then incubated for 72 hours at $25^{\circ} \mathrm{C}$ in k-elastin solution in water $(100 \mu \mathrm{g} / \mathrm{m} l)$. The incubated filters were washed extensively and air-dried. Micropore filters subjected to the above procedures without any elastin peptides and the elastin-coated filters are referred to as control filters (CF) and elastin-coated filters (EF), respectively. Treatment of EF with Lipoproteins and Anti-Elastin Antibody

$\mathrm{CF}$ and EF were incubated with 15\% human plasma LDL or $15 \%$ human plasma HDL in MEM or with the rabbit $\gamma$-globulin solutions (anti-human aortic elastin $\gamma$-globulin and control $\gamma$-globulin) in PBS at various protein concentrations at $4^{\circ} \mathrm{C}$ for 48 hours. After incubation, the filters treated with lipoproteins were washed with MEM and the filters treated with $\gamma$-globulin were washed with PBS. The dried, lipoprotein-treated filters and $\gamma$-globulintreated filters were placed in the Boyden chamber prior to assay. The abbreviations used for filters after these treatments are as follows: LDL-treated CF (LDL-CF), HDL-treated CF (HDL-CF), LDLtreated EF (LDL-EF), HDL-treated EF (HDL-EF), anti-elastin $\gamma$-globulin-treated CF (AEG-CF), control $\gamma$-globulin treated CF (CG-CF), anti-elastin $\gamma$ globulin-treated EF (AEG-EF), and control $\gamma$ globulin-treated EF (CG-EF).

\section{Preparation of Rat Platelet-Derived Attractant}

Blood from male Wistar rats, 6 to 12 weeks old, was added to a solution of $3.8 \%$ sodium citrate (9: 1 , vol $/ \mathrm{vol}$ ) and centrifuged at $200 \times \mathrm{g}$ for 10 minutes at room temperature to obtain platelet-rich plasma (PRP). Platelets obtained by centrifuging PRP at $1000 \times \mathrm{g}$ for 10 minutes at room temperature were suspended in PBS at a concentration of $2.5 \times 10^{8} / \mathrm{m} l$ and stored at $-80^{\circ} \mathrm{C}$. Before experiments, the platelets were freeze-thawed three times and $0.2 \mathrm{ml}$ of preparation, equivalent to $5 \times 10^{4}$ platelets, was introduced into the lower well of a Boyden chamber as platelet-derived attractant (PDA). The PDA in the platelet lysate was not identified in this study. Possible factors are PDGF ${ }^{12}$ ) and 12-HETE ${ }^{13)}$.

\section{Smooth Muscle Cells (SMC)}

The procedures for the isolation and culture of rat aorta cells have been described ${ }^{14)}$. SMCs were isolated from media explants of thoracic aortas from male Wistar rats, 6 to 8 weeks old. In culture, the cells began to grow and migrate out of the explants after 10 to 14 days. Cells were subcultured at a 1 to 2 split ratio when they became confluent and were maintained in MEM containing $10 \%$ FBS (10\% FBS/MEM). The cells showed multilayer growth and abundant SMC myofilaments. Cells after 5 to 10 subcultures were used for migration assays.

\section{Smooth Muscle Cell Migration}

Cell migration was assayed in a Boyden chamber as described previously ${ }^{1,15}$. Briefly, $0.2 \mathrm{~m} l$ of platelet homogenate and $0.6 \mathrm{~m} l$ of $10 \% \mathrm{FBS} / \mathrm{MEM}$ were introduced into the lower well beneath the filter, and a freshly prepared suspension of SMCs $\left(5 \times 10^{5}\right.$ per assay) in $10 \% \mathrm{FBS} / \mathrm{MEM}$ was introduced into the upper well. The chambers were incubated for 8 hours at $37^{\circ} \mathrm{C}$ under $5 \% \mathrm{CO}_{2}$ in air. The filter was then removed, fixed, stained with hematoxylin, and the number of cells in the filter (within $30 \mu \mathrm{m}$ of the upper surface) was determined by counting the 


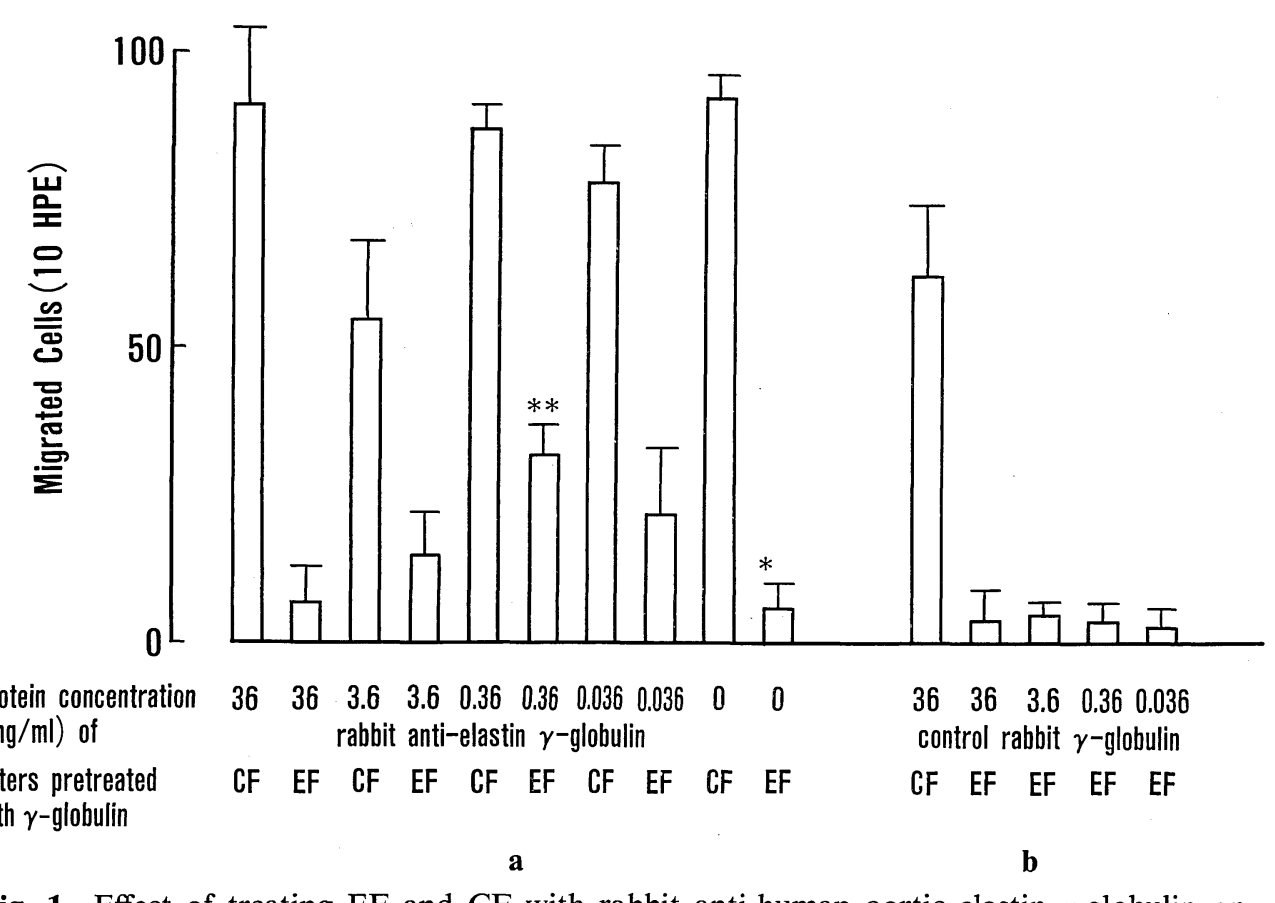

Fig. 1 Effect of treating EF and CF with rabbit anti-human aortic elastin $\gamma$-globulin on cultured rat aortic SMC migration. Cultured rat aortic SMCs $\left(5 \times 10^{5}\right)$ were put into the upper compartment and PDA into the lower compartment of a Boyden chamber. The CF or EF filters separating the two compartments were treated with PBS or rabbit anti-human aortic elastin $\gamma$-globulin (Fig. 1a) or control rabbit $\gamma$-globulin (Fig. $1 \mathrm{~b}$ ) at $4^{\circ} \mathrm{C}$ for 48 hours prior to the migration assay. The chamber was incubated at $37^{\circ} \mathrm{C}$ for 8 hours under $5 \% \mathrm{CO}_{2}$ in air as described in "Materials and Methods". $*<* *, \mathrm{p}<0.01$

number of nuclei in 10 high-power fields (HPF) $(\times 400)$. All assays were performed in triplicate and experiments were repeated at least three times. The magnitude of SMC migration is expressed as the average number of cells/10 HPF in separate experiments.

\section{Results}

1. Effect of Treating EF with Anti-Elastin $\gamma$ Globulin (AEG) on SMC Migration in Boyden Chambers

As shown in Fig. 1a, cultured rat aortic SMCs migrate well into a control filter $(\mathrm{CF})$ in response to the presence of rat PDA. This rat PDA-induced enhancement of migration is inhibited when the cells are tested in Boyden chambers with EF. However, SMCs again migrate well toward rat PDA in Boyden chambers when the EF are pretreated with antielastin $\gamma$-globulin (AEG-EF). The effect of the antibody does not depend on the dose of the antibody from $36 \mu \mathrm{g} / \mathrm{m} l$ to $36 \mathrm{mg} / \mathrm{ml}$. Antibodies at a dose of $360 \mu \mathrm{g} / \mathrm{ml}$ have the maximum effect on EF but do not eliminate all of the inhibitory effect. As shown in Fig. 1b, however, the same treatment of EF with the same dose range of normal rabbit $\gamma$-globulin has no significant effect.

2. Effect of Treating EF with LDL and HDL on SMC Migration in Boyden Chambers

Cultured rat aortic SMCs migrated as well in response to the presence of rat PDA in CF pretreated with LDL or HDL (LDL-CF, HDL-CF) as in CF (Fig. 2). The cells do not migrate in EF. However, the cells again migrate well in EF pretreated with $15 \%$ human plasma LDL (LDL-EF). In contrast, treatment of EF with $15 \%$ human plasma HDL does not change the SMC migration (Fig. 2).

\section{Discussion}

The migration of cultured rat aortic SMCs toward rat PDA in a Boyden chamber is completely inhibited when the chamber is fitted with the elastin-coated filter (EF) instead of a control filter (CF) ${ }^{1}$. . However, the cells again migrate well when the Boyden chambers are fitted with EF pretreated with human plasma LDL (LDL-EF, Fig. 2) or with rabbit anti-elastin $\gamma$-globulin (AEG-EF, Fig. 1a). This phenomenon is 


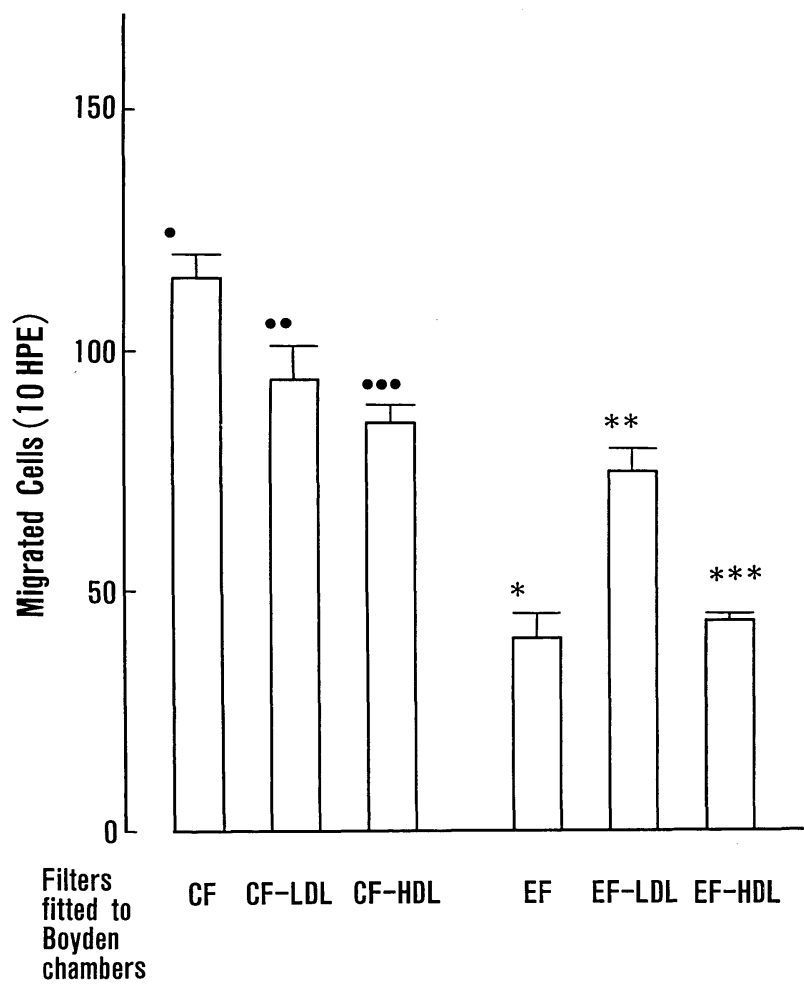

Fig. 2 Effect of treating EF and CF with LDL or HDL on cultured rat aortic SMC migration. Cultured rat aortic SMCs $\left(5 \times 10^{5}\right)$ were put into the upper compartment and PDA into the lower compartment of a Boyden chamber. The CF or EF filters separating the two compartments were treated with $15 \%$ human plasma LDL, $15 \%$ human plasma HDL, or PBS at $4^{\circ} \mathrm{C}$ for 48 hours prior to the migration assay. The chamber was incubated at $37^{\circ} \mathrm{C}$ for 8 hours under $5 \% \mathrm{CO}_{2}$ in air.

$*<* *, \mathrm{p}<0.01, \quad * * *<* *, \mathrm{p}<0.01, \quad \cdot<\cdot, \quad$ n.s., $\cdots<\cdot, \quad \mathrm{p}<0.01$

not observed when the Boyden chambers are fitted with HDL-EF or CG-EF, so that it is concluded that human plasma HDL and normal $\gamma$-globulin do not have the same effect as human plasma LDL and rabbit anti-elastin $\gamma$-globulin. Anti-elastin antibody has a high affinity for elastin. LDL also has a high affinity for elastin ${ }^{4-6)}$; thus, the binding of specific proteins to elastin mask the cell biological activity of elastin.

The amino acid composition of the elastin used in the present study does not differ from that of elastin peptides previously reported ${ }^{9}$. The rabbit anti-elastin $\gamma$-globulin described above reacts well with k-elastin, but not with connective tissue components other than elastin, such as Types I, III, and $\mathrm{V}$ collagens on immunoblotting (see Materials and Methods). The antibody also stains only the elastic fibers in the paraffin sections of human artery. As shown previously1), the inhibition of SMC migration by $\mathrm{EF}$ arises from the elastin molecule attached to the filter. The present data confirm that this assumption is correct.

Although the mechanism by which elastin molecules attached to EF inhibit cultured rat aortic SMC migration in response to rat PDA is not known, an interaction between the Val-Gly-Val-Ala-Pro-Gly(VGVAPG) sequence in the hydrophobic region of elastin and cell membrane associated proteins ${ }^{15}$ ) might be involved. Since the binding of LDL cholesterol with elastin is related to the hydrophobic sequence of elastin ${ }^{16)}$, LDL pretreatment may mask the active site of elastin responsible for the inhibition of SMC migration. HDL also has an affinity for elastin, although less than that of $\mathrm{LDL}^{4}$ ) ; however, HDL does not eliminate the inhibition of cell migration when used to treat EF prior to the migration assay (Fig. 2). The reason why the two types of lipoprotein, both with affinities for elastin, behave differently in terms of SMC migration in vitro is not known at present.

In conclusion, LDL has been found deposited along with elastic fibers in atherosclerotic lesions?) and is usually included among the atherogenic factors in blood. The results of the present study indicate that part of the atherogenic effect of LDL might be due to its binding effect on elastin. The deposition of LDL in intimal elastic fibers in vivo may foster medial SMC migration toward the intima.

\section{References}

1) Ooyama, T., Fukuda, K., Oda, H., Nakamura, H. and Hikita, Y.: Substratum-bound elastin peptides inhibit aortic smooth muscle cell migration in vitro. Arteriosclerosis, 7: 593-598 (1987).

2) Soskel, N. T., Frank, T. V. and Hoidal, J. R.: Elastin substratum inhibits proliferation of neonatal rat aortic smooth muscle cells. Am. Rev. Res., 133: 129 (1988).

3) Grande, J. G., Davis, H. R., Bates, S., Mathews, M. B. and Glagov, S.: Effect of an elastin growth substrate on cholesteryl ester synthesis and foam cell formation by cultured aortic smooth muscle cells. Atherosclerosis, 68: 87-93 (1987).

4) Noma, A., Hirayama, T. and Yachi, A.: Studies on the binding of plasma low density lipoproteins to arterial elastin. Connective Tissue Res., 11: 123-133 (1983).

5) Podet, E. J., Schaffer, D. R., Gianturco, S. H., Bradley, W. A., Yang, C. Y. and Guyton, J. R.: Interaction of low density lipoproteins with human aortic elastin. Arteriosclerosis and Thrombosis 11: 116-122 (1991).

6) Tokita, K., Kanno, K. and Ikeda, K.: Elastin sub- 
fractions as binding site for lipids. Atherosclerosis, 28: 111-119 (1977).

7) Kramsch, D. M. and Hollander, W.: The interaction of serum and arterial lipoproteins with elastin of the arterial intima and its role in the lipid accumulation in atherosclerotic plaques. J. Clin. Invest., 52: 236247 (1973).

8) Robert, L., Robert, B. and Robert, A. M.: Molecular biology of elastin as related to ageing and atherosclerosis. Exp. Gerontol., 5: 339-356 (1979).

9) Giro, W. G. and Davidson, J. M.: Elastin. Methods in Enzymol., 16: 656-673 (1982).

10) Ooyama, T., Fukuda, K., Masuda, S. and Nakamura, H.: Administration of elastase blocks the formation of fragmented elastic fibers in aorta of rabbit. Artery, 16: 293-311 (1989).

11) Havel, R. J., Eder, H. A. and Bragdon, J. H.: The distribution and chemical composition of ultracentrifugally separated lipoproteins in human serum. J. Clin. Invest., 34: 1345-1351 (1955).

12) Grotendorst, G. R., Seppa, H. E. J., Kleinman, H. K. and Martin, G. R.: Attachment of smooth muscle cells to collagen and their migration toward plateletderived growth factor. Proc. Natl. Acad. Sci. U.S.A., 78: 3669-3672 (1981).

13) Nakao, J., Ooyama, T., Chang, W. C., Murota, S. and Orimo, H.: Platelets stimulate aortic smooth muscle cell migration in vitro-Involvement of 12L-hydroxy-5,8,10,14-eicosatetraenoic acid. Atherosclerosis, 43: 143-150 (1982).

14) Ross, R.: The smooth muscle cell. II. Growth of smooth muscle in culture and formation of elastic fibers. J. Cell Biol., 50: 172-186 (1975).

15) Mecham, R. P., Hinek, A., Entwiste, R., Wrenn, D. S., Griffin, G. L. and Senior, R. M.: Elastin binds to a multifunctional 67 kilodalton peripheral membrane protein. Biochemistry, U.S.A., 28: 3716-3726 (1989).

16) Hornebeck, W. and Partridge, S. M.: Conformational changes in fibrous elastin due to calcium ions. Eur. J. Biochem., 51: 73-79 (1973).

\section{Summary}

Cultured rat aortic smooth muscle cells (cultured rat aortic SMCs: $5 \times 10^{5}$ ) suspended in the upper compartment of a Boyden chamber migrated through the pores of the filters separating the two compartments in response to rat platelet-derived attractant (rat PDA) in the lower compartment. We have previously demonstrated that this enhanced migration is inhibited when the filter is coated with elastin purified from normal human aorta. However, cultured rat aortic SMCs again migrate well toward the rat PDA when the elastin-coated filter is treated prior to the migration assay with $15 \%$ human plasma LDL. Pretreatment of elastin-coated filters with $15 \%$ human plasma HDL fails to remove the inhibitory effect of elastin. Pretreatment of elastin-coated filters with rabbit-anti human aortic elastin antibody at protein concentrations of from $36 \mu \mathrm{g} / \mathrm{ml}$ to $36 \mathrm{mg} / \mathrm{m} l$ also removed the inhibitory effect of elastin, although to the lesser extent than the effect of LDL.

These results suggest that the atherogenic effect of LDL might be due in part to an effect in vivo on the elastin, elastic fibers of the arterial wall.

Key words: Elastin, Smooth muscle cell migration, LDL, Atherosclerosis. 